\title{
ARTÉRIA HEPÁTICA: EMBRIOLOGIA, ANATOMIA E IMPLICAÇÕES NO TRANSPLANTE HEPÁTICO
}

\author{
Hepatic artery: embryology, anatomy and liver transplantation implications
}

\author{
Olival Cirilo Lucena da Fonseca-Neto', Beatriz Rezende Monteiro²
}

\begin{abstract}
RESUMO
Introdução: A artéria hepática (AH) surge a partir do tronco celíaco (TC), com origem na aorta, porém determinadas mudanças em diferentes estágios do desenvolvimento humano no período embrionário podem gerar diversas modificações na conformação normal da vasculatura. O conhecimento da distribuição arterial tanto normal como anômala é fator crucial para o sucesso de cirurgias complexas como o transplante hepático, evitando complicações vasculares que possam levar à perda do enxerto. Objetivo: Realizar uma revisão da literatura a fim de contribuir com o conhecimento dos profissionais e estudantes da área, e preservar indivíduos que necessitam desses procedimentos. Métodos: Foi realizada revisão de literatura no PUBMED. Os descritores em inglês utilizados foram: "hepatic artery", "liver transplantation" e "anatomy". Resultados O suprimento sanguíneo hepático na embriogênese, é realizado pela artéria gástrica esquerda, artéria hepática comum e artéria mesentérica superior. A anatomia da irrigação hepática é bastante diversificada, o que dificulta a avaliação radiológica, angiográfica e cirúrgica, caso não haja um bom conhecimento da anatomia clássica e anômala por parte da equipe médica. A classificação de Hiatt é simples e prática, utilizando apenas seis tipos de variações ao invés de 10, como Mitchels. A avaliação cautelosa das estruturas vasculares e biliares anterior ao transplante é de extrema importância para prevenção de complicações, como a trombose da artéria hepática. Conclusão: O impacto das complicações vasculares é enorme e, por isso, é fundamental que os cirurgiões tenham conhecimento pleno da anatomia vascular e suas variações, a fim de reduzir, gradativamente, a incidência de complicações.
\end{abstract}

Descritores: Artéria Hepática; Anatomia; Transplante hepático

\footnotetext{
Instituições:

1 Serviço de Cirurgia Geral e Transplante de Fígado do Hospital Universitário Oswaldo Cruz, Recife/PE, Brasil.

2 Curso de Medicina do Centro Universitário Maurício de Nassau, Recife/PE, Brasil.
}

Correspondence:

Olival Cirilo Lucena da Fonseca Neto

E-mail: olivalneto@globo.com

https://doi.org/10.53855/bjt.v24i1.005

Recebido em: 02/08/2020

Aceito em: 22/02/2021

\section{INTRODUÇÃO}

Desde 500 anos A.C., a partir de Alcméon de Crotona, responsável pelas primeiras dissecções em animais, o estudo da anatomia é reconhecido como fator fundamental para a realização de procedimentos

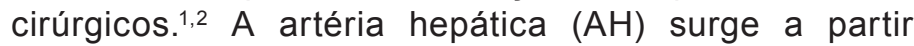
do tronco celíaco (TC), com origem na aorta, que se ramifica em artéria gástrica esquerda (AGE), artéria esplênica $(A E)$ e artéria hepática comum (AHC). Esta, após emergência da artéria gastroduodenal, continua como artéria hepática própria e ramifica-se em artéria hepática direita e artéria hepática esquerda no hilo hepático. 
Determinadas mudanças em diferentes estágios do desenvolvimento humano no período embrionário podem gerar diversas modificações na conformação normal da vasculatura. ${ }^{1,3} \mathrm{Em} \mathrm{1966,} \mathrm{o} \mathrm{clássico} \mathrm{estudo} \mathrm{de}$ Mitchels definiu as múltiplas variações anatômicas da AH modificadas por Hiatt, em 1994, organizando-as em apenas seis categorias, ao invés de 10 . A presença de variações anatômicas na $\mathrm{AH}$ varia de $20 \%$ a $50 \%$ na literatura, ${ }^{4}$ sendo relativamente frequente e, por isso, o conhecimento da distribuição arterial, tanto normal como anômala, é um fator crucial para o sucesso de cirurgias complexas, como o transplante hepático, a fim de evitar possíveis danos ou complicações vasculares que possam levar à perda do enxerto.

As complicações vasculares não são incomuns dentro do transplante de fígado, com incidência de até $20 \%$ em alguns estudos, apesar de claros avanços em técnicas cirúrgicas vasculares. ${ }^{5,6}$ Dentre os principais resultados desfavoráveis que podem ocorrer, podemos destacar o aneurisma, estenose e trombose da artéria hepática, além das possíveis complicações biliares, entre outras.

\section{OBJETIVO}

Diante da relevante incidência das variações no sistema arterial hepático e da sua influência em cirurgias que envolvem a região hepática, principalmente o transplante, torna-se fundamental a realização de uma revisão da literatura, a fim de contribuir com o conhecimento dos profissionais e estudantes da área, e preservar indivíduos que necessitam desses procedimentos, evitando as respectivas complicações tanto na captação quanto no implante hepático e diminuindo a alta mortalidade associada.

\section{MÉTODOS}

Foi realizada revisão de literatura por meio de busca na base eletrônica de dados PUBMED. Os descritores em inglês utilizados foram: "hepatic artery", "liver transplantation" e "anatomy". A busca foi limitada a artigos publicados em inglês e em português, estudos com seres humanos, população alvo com indivíduos adultos, sem delimitações para tipos de estudos. O período avaliado foi a partir do ano 2000 até o mês de junho de 2018. Estudos adicionais de relevância para a discussão foram incluídos.

\section{RESULTADOS}

\section{Embriologia}

O contexto embriológico é de extrema importância, principalmente, para o entendimento das variações anatômicas existentes e suas implicações. ${ }^{3}$ A partir da formação da placa hepática reconhecida como o espessamento do endoderma pertencente ao intestino primitivo, na $4^{\text {a }}$ semana de vida intraútero, o primórdio do fígado se desenvolve em direção ao tecido conectivo mesenquimal do septo transverso (Figuras 1 e 2), responsável pela origem das ilhotas formadoras de células sanguíneas. Paralelamente a esse evento, a aorta dorsal e a porção ventral que estão interligadas por uma artéria ventral anastomótica que regride e desaparece posteriormente, começam a se fundir em direção à linha média. Contudo, os seguimentos arteriais ventrais 10 , 11 e 12 não regridem completamente, sofrendo apenas um retrocesso e formando respectivamente, a artéria gástrica esquerda (AGE), artéria esplênica ( $A E)$ e artéria hepática comum $(\mathrm{AHC})$. $^{3,7-9} \mathrm{~A}$ ausência do tronco celíaco pode ser detectada através de estudos de imagem e de dissecções anatômicas, tendo uma incidência rara correspondendo a $0.1-2.6 \%$ no mundo..$^{8,9,10}$

Figura 1 - Corte transversal do embrião, evidenciando a formação da placa hepática em direção ao septo transverso (desenho esquemático realizado pelos autores).

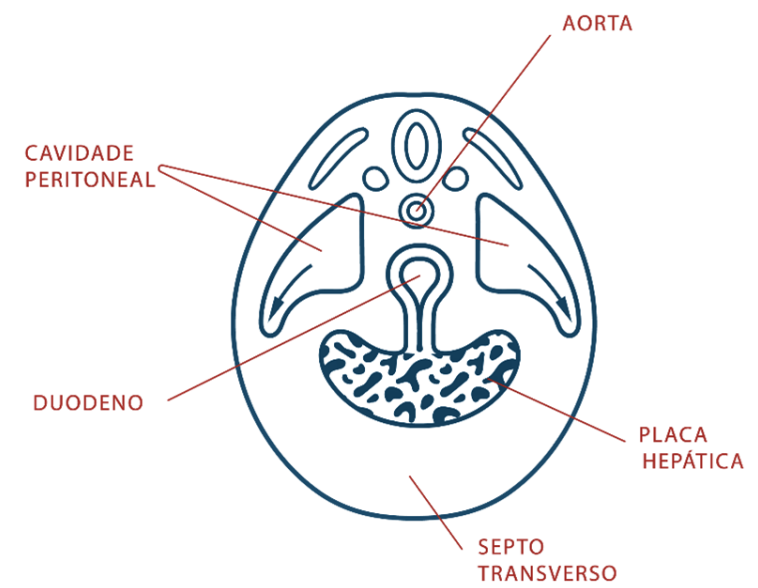

Figura 2 - Corte transversal do embrião após expansão da cavidade peritoneal e com fígado em desenvolvimento (desenho esquemático realizado pelos autores).

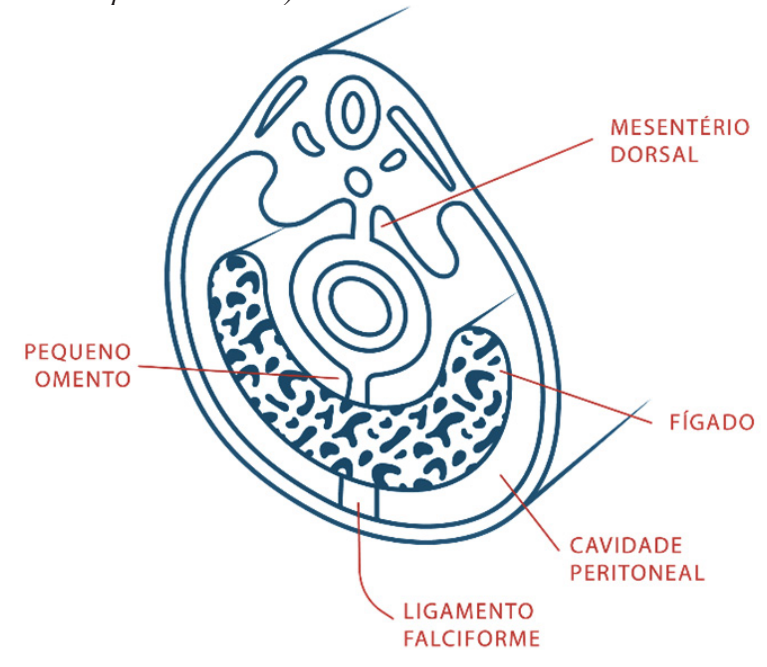


Dessa forma, o suprimento sanguíneo hepático na embriogênese (Figura 3) é realizado pela AGE, responsável pelo segmento lateral esquerdo; a $\mathrm{AHC}$, pelos segmentos paramedianos, e artéria mesentérica superior (AMS), oriunda do $13^{\circ}$ ramo da aorta ventral, e responsável pelo segmento lateral hepático direito. As artérias gástricas esquerda e mesentérica superior, nesse contexto, são reconhecidas como artéria hepática esquerda embrionária e artéria hepática direita embrionária. ${ }^{1,3,7}$

Figura 3 - Corte mediano da metade caudal de um embrião no final da quinta semana (desenho esquemático realizado pelos autores)

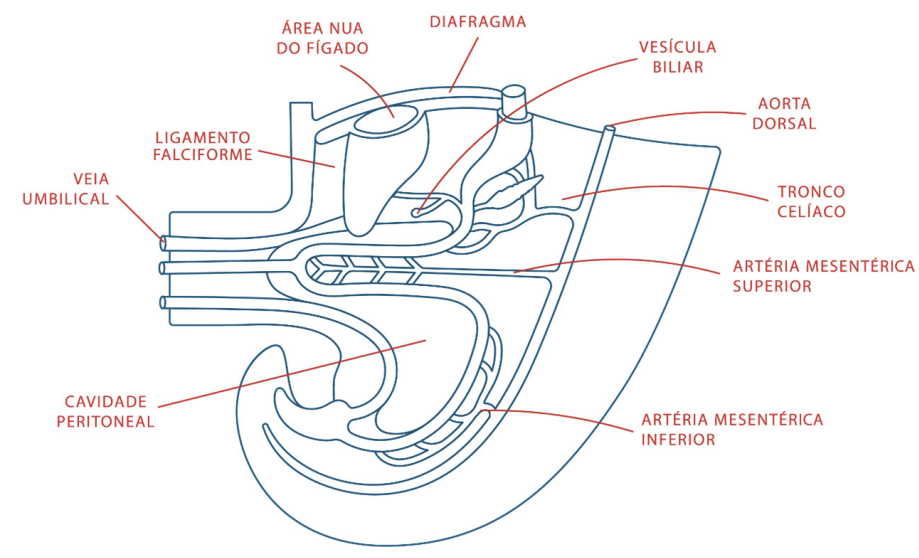

Ao final da $8^{a}$ semana de desenvolvimento intraútero, as artérias responsáveis pela irrigação do fígado estão todas anastomosadas no hilo hepático. As variações anatômicas podem surgir devido ao regresso parcial da artéria ventral anastomótica e/ou de alterações dos padrões comuns de retrocesso dos ramos ventrais. ${ }^{7}$

\section{Anatomia}

O estudo da artéria hepática $(\mathrm{AH})$ teve início na Antiguidade, a partir de grandes estudiosos como Aristóteles e Galeno. No entanto, sua anatomia foi detalhadamente descrita apenas através de Jacques Benigne Winslow e Albert Haller, considerados pais da angiologia moderna, que definiram a anatomia correta do TC. ${ }^{2,4,6}$

$\mathrm{Na}$ anatomia clássica, que serve de guia para compreensão do suprimento vascular, o fígado é suprido com sangue arterial pela artéria hepática própria (AHP), continuação da $A H C$ que é um dos principais ramos do TC. A AHP penetra no ligamento hepatoduodenal, juntamente com a veia porta do fígado e o ducto colédoco, em direção ao hilo hepático, logo após a emergência da artéria gástrica direita. A AHP se divide em dois ramos - esquerdo e direito - para cada lobo hepático, sendo a partir dessa divisão, reconhecida como artéria hepática direita (AHD) e artéria hepática esquerda (AHE).

A anatomia da irrigação hepática é bastante diversificada, o que dificulta a avaliação radiológica, angiográfica e cirúrgica, caso não haja bom conhecimento da anatomia clássica e anômala por parte da equipe médica. Em casos de variação anatômica, os lobos hepáticos podem ser supridos por outros vasos que podem atuar como acessórios em adição ao suprimento normal ou como substitutos. ${ }^{4}$ É possível, por exemplo, que a AMS irrigue o lobo hepático direito ou que a AGE irrigue o lobo hepático esquerdo. Sendo assim, múltiplas variações anatômicas foram detectadas e classificadas, em 1966 por Mitchel, e, posteriormente, em 1994, sofrendo alterações por Hiatt, classificação mais utilizada na atualidade.

A classificação de Hiatt é simples e prática, utilizando apenas seis tipos de variações, ao invés de 10, como Mitchels. ${ }^{4}$ Hiatt determinou que o tipo lé sempre referente a anatomia normal; o tipo II é quando a AHE é ramo da AGE ou a AHE é acessória à AGE; o tipo III, quando a AHD é ramo da AMS ou AHD é acessória à AMS; o tipo IV, quando há associação entre a anatomia normal(tipo I) e a variação do tipo II ou a AHE é acessória à AGE e a AHD é acessória à AMS ou, ainda, a AHE é acessória à $A G E$ e a $A H D$ é ramo da $A M S$; o tipo $V$, quando a $\mathrm{AHC}$ é ramo da AMS; e o tipo $\mathrm{VI}$, quando a $\mathrm{AHC}$ é ramo direto da aorta. É possível comparar a classificação de Mitchels e Hiatt, através da tabela 1.

Tabela 1 - Classificação da distribuição arterial hepática proposta por Mitchel(1966) e Hiatt (1994)

\begin{tabular}{|c|c|c|}
\hline ANATOMIA ARTERIAL HEPÁTICA & $\begin{array}{c}\text { Classificação } \\
\text { de Mitchel }\end{array}$ & $\begin{array}{c}\text { Classificação } \\
\text { de Hiatt }\end{array}$ \\
\hline Anatomia normal & TIPO I & TIPO II \\
\hline AHE ramo da AGE & TIPO II & TIPO ॥ \\
\hline AHD ramo da MAS & TIPO III & TIPO III \\
\hline AHE ramo da AGE + AHD ramo da AMS & TIPO IV & TIPO IV \\
\hline AHE acessória da AGE & TIPO V & TIPO II \\
\hline AHD acessória da MAS & TIPO VI & TIPO III \\
\hline AHE acessória da AGE + AHD acessória AMS & TIPO VII & TIPO IV \\
\hline AHE acessória da AGE + AHD ramo AMS & TIPO VIII & TIPO IV \\
\hline AHC ramo da MAS & TIPO IX & TIPO V \\
\hline AHD e AHE ramos da AGE & TIPOX & - \\
\hline AHC ramo da aorta & - & TIPO VI \\
\hline $\begin{array}{l}\text { "- }=\text { variação não presente } ; \\
\text { AHE = artéria hepática esquerda } ; \\
A G E=\text { artéria gástrica esquerda } ; \\
\text { AHD = artéria hepática direita; } \\
A M S=\text { artéria mesentérica superior; } \\
\text { AHC }=\text { artéria hepática comum. }\end{array}$ & & \\
\hline
\end{tabular}


Dentre as variações descritas por Hiatt, a mais comumente observada é a tipo III, seguida por tipo II e IV. As outras classificações são raras na literatura, o que comprova que o desenvolvimento embriológico da aorta é um processo complexo e multifatorial. ${ }^{4,11,12}$ Além dos tipos citados anteriormente, ainda existem variações não classificáveis. Koops et al ${ }^{13}$ descreveram uma ocorrência de $1,8 \%$ de apresentações raras. Alakamm et al ${ }^{7}$ relataram um caso raro em que todo o suporte arterial hepático era derivado da AMS, sendo a AHC completamente inexistente, encontrado em $0,3 \%$ de alguns estudos com amostras significativas.

\section{Implicações no transplante hepático}

O conhecimento da distribuição normal e anômala dos vasos sanguíneos é fator crucial para o sucesso de procedimentos cirúrgicos, inclusive o transplante hepático, que é uma cirurgia complexa e desafiadora, e essencial para a sobrevivência de pacientes hepatopatas em estágio terminal. Sendo assim, a avaliação cautelosa das estruturas vasculares e biliares anterior ao transplante é de extrema importância para prevenção de complicações, como o retransplante, as complicações biliares, estenose da $\mathrm{AH}$ e trombose da artéria hepática (TAH). ${ }^{14,15}$

Problemas vasculares, apesar de grandes progressos em técnicas cirúrgicas, ainda têm incidência de 0,8\% a $20 \%$, e põem em risco o sucesso do transplante, representando importante causa de morbimortalidade, com frequência na literatura que varia de $11 \%$ a $60 \% .^{5,14}$

A TAH se mantém como a mais comum e fatal complicação vascular após o transplante de fígado, aumentando de forma devastadora a mortalidade dos pacientes submetidos a esse procedimento. Devido às frequentes anastomoses nas reconstruções arteriais hepáticas, o risco de TAH aumenta consideravelmente, com incidência que varia na literatura de $2 \%$ a $15 \% .^{15,16}$

A TAH pode ser desenvolvida de forma precoce ou tardia. A forma precoce se dá em menos de 30 dias após o transplante e está muito associada com uma história natural mais agressiva, associada a elevados índices de perda do enxerto e de mortalidade dos pacientes. Em comparação com a TAH precoce, a tardia ocorre após os primeiros 30 dias desse procedimento e está relacionada com um curso clínico menos agressivo. ${ }^{15}$
Depois da disfunção primária do enxerto, a TAH é a segunda principal causa de falha do enxerto e a primeira causa de perda do enxerto e mortalidade, com índices de $33 \%$ no pós-operatório imediato. Sua etiologia ainda não é bem estabelecida, mas é possível associála a alguns fatores como erros de técnica cirúrgica (responsável por $20 \%$ dos casos), variações anatômicas arteriais hepáticas de complicada reconstrução, torção arterial e disparidade no diâmetro entre as artérias do enxerto e do receptor ou na viabilidade e qualidade desses vasos. ${ }^{13-15}$

Anormalidades anatômicas podem estar relacionadas ao aumento dos índices de TAH e as mais frequentemente encontradas são as variações extra-hepáticas, com incidência de 20-50\%.4,10,16 As reconstruções do backtable são consideradas fator de risco para desenvolvimento de $\mathrm{TAH}^{15,17}$ Mas, outros estudos ${ }^{16,18}$ sugerem que não há relação entre reconstruções arteriais e o aumento da incidência de TAH, e por esse motivo, deve haver fatores concomitantes ainda não determinados que provoquem essa complicação. Além disso, também se associa significantemente TAH precoce com transfusão sanguínea no pós-operatório e tempo de operação prolongado. ${ }^{15}$

$\mathrm{Na}$ atualidade, através dos avanços das técnicas endovasculares intervencionistas, grande parte dos pacientes tem recebido tratamento, apresentando menos morbimortalidade em relação ao retransplante.15 Kazemi et al ${ }^{6}$ recomenda o método de transposição arterial esplênica para reconstrução arterial, que proporciona melhor exposição e melhores resultados em relação a tempo de operação e índices de sangramento e, consequentemente, menor exposição a possibilidade de desenvolvimento de complicações associadas.

\section{CONCLUSÃO}

Tanto as variações mais comuns da anatomia arterial hepática quanto as mais raras requerem diferentes desafios em relação às técnicas cirúrgicas. Considerando nesse sentido, a escassez de enxertos disponíveis em relação à demanda existente, o impacto das complicações vasculares é enorme, e por isso, é fundamental que os cirurgiões tenham conhecimento pleno da anatomia vascular e suas variações, a fim de reduzir gradativamente a incidência de complicações como a TAH. 


\section{ABSTRACT}

Introduction: The hepatic artery ( $\mathrm{HA}$ ) arises from the celiac trunk $(\mathrm{CT})$, originating from the aorta, but certain changes, in different stages of human development in the embryonic period, may generate several changes in the normal conformation of the vasculature. Knowledge of both normal and anomalous arterial distribution is a crucial factor for the success of complex surgeries, such as liver transplantation, avoiding vascular complications that may lead to graft loss. Purpose: To review the literature in order to contribute to the knowledge of professionals and students in the area and to preserve the individuals who need these procedures. Methods: A literature review was performed at PUBMED. The English descriptors used were: 'hepatic artery', 'liver transplantation' and 'anatomy'. Results: Hepatic blood supply in embryogenesis is performed by the left gastric artery, the common hepatic artery and the superior mesenteric artery. The anatomy of hepatic irrigation is quite diverse, which makes the radiological, angiographic and surgical evaluation difficult if there is not a good knowledge of the classical and anomalous anatomy by the medical team. Hiatt's classification is simple and practical, using only 6 types of variations instead of 10 as Mitchels. Careful assessment of vascular and biliary structures prior to transplantation is of utmost importance for the prevention of complications such as hepatic artery thrombosis. Conclusion: The impact of vascular complications is enormous and it is therefore essential that surgeons have full knowledge of vascular anatomy and its variations in order to gradually reduce the incidence of complications.

Keywords: Hepatic Artery; Anatomy; Liver transplant.

\section{REFERÊNCIAS}

1. Sebben GA, Rocha SL, Sebben MA, Parulosso FP, Gonçalves $\mathrm{BHH}$. Variações da artéria hepática: estudo anatômico em cadáveres. Rev Col Bras Cir. 2013;40(3):221-6.

2. Cunha AG, Bastos JLA, Meyer-Nascimento RJ. Transplante hepá-tico: aspectos históricos. JBT J Bras Transpl. 2013;16(1):1715-41.

3. Zagyapan R, Kurkcuoglu A, Bayraktar A, Pelin C, Aytekin C. Anatomic variations of the celiac trunk and hepatic arterial system with digital subtraction angiography. Turkish J Gastroenterol. 2015;25:104-9.

4. Fonseca-neto OCL, Lima HCS, Rabelo P, Melo PSV, Amorim AG, Lacerda CM. Anatomic variations of hepatic artery: a study in 479 liver transplantations. ABCD Arq Bras Cir Dig. 2017;30(1):35-7.

5. Frongillo F, Lirosi MC, Nure E, Inchingolo R, Bianco G, Silvestrini $\mathrm{N}$ et al. Diagnosis and management of Hepatic Artery complications after liver transplantation. 2015;47:2150-5.

6. Kazemi K, Samidoost P, Deilami HN, Hosseini SAM, Nikeghbalian $S$, Shamsaeefar A et al. A new consideration in hepatic artery reconstruction in adult liver transplant: arterial transposition versus extra-anatomic jump grafts. Exp Clin Transplant. 2017;1:204-7.

7. Alakkam A, Hill RV, Saggio G. Superior mesenteric origin of the proper hepatic artery: embryological and clinical implications. Surg Radiol Anat. 2016;38(6):747-50.

8. Venieratos D, Panagouli E, Lolis E, Tsaraklis A, Skandalakis P. A morphometric study of the celiac trunk and review of the literature. Clin Anat. 2013;26(6):741-50.

9. Zagyapan R, Kurkçuoglu A, Bayraktar A, Pelin C, Aytekin C. Anatomic variations of the celiac trunk and hepatic arterial system with digital subtraction angiography. Turk J Gastroenterol. 2014; 25(1):104-9.
10. Freitas ACT, Coelho JCU, Matias JEF, Neto CZ, Martins EL, Druszez CC. Anatomia arterial hepática: estudo em 150 transplantes hepáticos. Rev Col Bras Cir, 2000;28(1):13-6.

11. Ugurel MS, Battal B, Bozlar U, NUral MS, Tasar M, Ors F et al. Anatomical variations of hepatic arterial system, coeliac trunk and renal arteries: ananalysis with multidetector CT angiography. Br J Radiol. 2010; 83:661-7.

12. Andraus W, Haddad LBP, Ducatti L, Martino RB, Santos VR, D'albuquerque LAC. Reconstrução arterial no transplante hepático: a melhor reconstrução para variação da artéria hepática direita. ABCD Arq Bras Cir Dig. 2013;26(1):62-5.

13. Koops A, WojciechowskiB, Broering DC, Adam G, Krupski-Berdien G. Anatomic variations of the hepatic arteries in 604 selective celiac and superior mesenteric angiographies. SurgRadiolAnat. 2004;26:239-44.

14. Özbilgin M, Ünek T, Egeli T, Agalar C, Ozkardesler S, Obuz F. Hepatic artery reconstruction with autologous inferior mesenteric artery graft in liver donor liver transplant recipients. Transplant Proc. 2017;49:566-70.

15.Zhicheng Xue, Maogen Chen, Xuzhi Zhang, Guodong Wang, Xiaoshun He, Linwei Wu et al. ANZ J Surg, 2018;88(3):172-6.

16. Reigada CHP, Ataide EC, Mattosinho TAP, Boin IFSF. Hepatic artery thrombosis after liver transplantation: five-year experience at the State University of Campinas. Transplant Proc. 2017;49:86770.

17. Kornasiewicz O, Dudek K, Lewandowski Z, Grat ZM, Scibisz A, Nyckowski P et al. Low incidence of hepatic artery after hepatic artery reconstruction during liver transplantation. Transplant Proc. 2009;41:3138-40.

18. Gunsar F, Rolando N, Pastacaldi S, Patch D, Raimondo ML, Davidson $\mathrm{B}$ et al. Late hepatic artery thrombosis after orthotopic liver transplantation. Liver Transplant. 2003;9(6):605-11. 